\title{
Assessment of Shoulder Mobility and Stability in Reverse Total Shoulder Arthroplasty: a Sensor Perspective
}

\author{
Matthias A Verstraete, $\mathrm{PhD}^{1 *}$; Michael A Conditt, $\mathrm{PhD}^{1}$; \\ Moby I. Parson, MD $^{2}$; Joseph DeCerce ${ }^{1}$; Gordon Goodchild ${ }^{1}$; \\ Alex Greene ${ }^{3}$, Chris Roche ${ }^{3}$ \\ ${ }^{1}$ OrthoSensor Inc., Fort Lauderdale, Florida, USA \\ ${ }^{2}$ Portsmouth Hospital, Portsmouth, New Hampshire, USA \\ ${ }^{3}$ Exactech, Gainesville, Florida, USA \\ Matthias.Verstraete@orthoSensor.com
}

\begin{abstract}
In reverse total shoulder arthroplasty stability is primarily controlled by the soft-tissue constraints rather than the congruency of the articulating surfaces. However, assessing the tension in these stabilizing structures currently remains highly subjective intraoperatively. In an attempt to quantify this feel during surgery, an intra-articular load sensor is introduced in this paper. In an in-vitro setting, the load sensor was used in eight reverse total shoulder arthroplasties on full torso specimens. The specimens were mounted on an inclined chair, such that the scapula could freely move to mimic surgical conditions. The resulting load vectors were captured through the range of motion under three different conditions, subjectively categorized by the surgeon as having a low, normal and high tightness. In neutral rotation and under a subjectively optimal condition assessed as neither too tight nor too loose, glenohumeral loads in the range of 10-20lbs were observed. For the same subjectively assessed optimal shoulder, loads up to $30 \mathrm{lbs}$ were observed under maximum internal/external humeral rotation. This contrasts the subjectively assessed loose and tight conditions, where lower resp. higher loads were observed that additionally affected the range of motion. On the other hand, stability was potentially missing under loose conditions as no increase in load was observed near the limits of the range of motion, indicating the lack of soft tissue restraint. In conclusion, this series of invitro experiments has shown the relevance and potential clinical value of assessing a
\end{abstract}


shoulder's stability and mobility using intra-articular load measurements during the trialing phase.

\section{Introduction}

Whereas pain relief is a primary objective of total shoulder arthroplasty, patients' perception of their post-operative functioning is additionally affected by the mobility and stability of their replaced joint. In reverse total shoulder arthroplasty (rTSA), it is well understood that the latter is primarily controlled by the soft-tissue constraints rather than the congruency of the articulating surfaces (Elmore, 2013) (Veeger, 2007). However, assessing the tension in these stabilizing structures currently remains highly subjective and relies on the surgeons' feel and experience intra-operatively. In an attempt to quantify this feel during surgery, an intra-articular load sensor is used in this paper.

While past research has provided insight into the loads occurring across the shoulder joint during daily activities (Westerhoff, 2009), limited work is available indicating the glenohumeral load magnitudes and patterns during passive assessments that are typically performed intra-opeatively during trialing. Therefore, this paper presents the results from a series of in-vitro experiments measuring the intra-articular shoulder loads through the range of motion while mimicking surgical conditions of reverse total shoulder arthroplasty.

\section{Methods}

A wireless sensor has been developed that is dimensionally equivalent to a standard humeral trial for rTSA (Figure 1). This sensor measures the intra-articular loads using three capacitive sensors embedded in the articulating surface. Wireless communication enables real-time display of the threedimensional load vector and load magnitude in the glenohumeral joint during component trialing in rTSA.

In an in-vitro setting, this sensor was used in eight reverse total shoulder arthroplasties on full torso specimens. The specimens were mounted on an inclined chair, such that the scapula could freely move to mimic surgical conditions. The resulting load vectors were captured through the range of motion under three different conditions, subjectively categorized by the surgeon as having a low, normal and high tightness.

\section{Results}

For the tested shoulder specimens, the newly developed sensor provided insight into the load magnitude and characteristics through the range of motion (Figure 2). In neutral rotation and under a subjectively optimal condition assessed as neither too tight nor too loose, glenohumeral loads in the range of 10-20lbs were observed. For the same subjectively assessed optimal shoulder, loads up to $30 \mathrm{lbs}$ were observed under maximum internal/external humeral rotation. When observing the subjectively loose condition, the loads were remarkably lower, particularly near the limits of the range of motion. This resulted in an increased range of motion resulting in a potentially unstable condition, since a limited load increase was observed under maximum external rotation (a measure of the passive resistance to dislocation). In contrast, higher loads and a reduced range of motion were observed under tight conditions, with a pronounced increase of the loads near maximum internal/external rotation. 


\section{Discussion}

In an in-vitro setting, sensor technology enabled the assessment of intra-articular loads during the trialing phase of reverse total shoulder arthroplasty. The reported load ranges were in line with the limited literature that is available on the subject (Higa, 2018). In addition, these in-vitro experiments allowed the identification of characteristic load patterns through the range of motion. These load patterns indicate the relevance of dynamic testing, as the surgeons' subjective assessment of shoulder tightness poorly correlated with the loads under neutral rotation. Only by exploring the limits of the range of motion, a difference in load magnitude was observed between a loose and a tight condition. Whereas higher loads are indicative of increased stability, this occurs concomitant with a change in the shoulders' mobility. Under maximum internal/external rotation, loads exceeding $40 \mathrm{lbs}$ indicated a reduction in the range of motion. On the contrary, the absence of a load increase towards maximum internal/external rotation is believed to indicate a potential risk for instability as soft tissue constraints to the shoulder mobility are lacking. As such, we believe that the use of sensor technology during rTSA has the potential to address instability as a dominant cause for revision surgery (Chae, 2018).

\section{Conclusion}

In conclusion, this series of in-vitro experiments has shown the relevance and potential clinical value of assessing a shoulder's stability and mobility using intra-articular load measurements during the trialing phase. Future steps include a clinical series during which these intra-articular measurements are linked to functional and subjective patient reported outcomes.

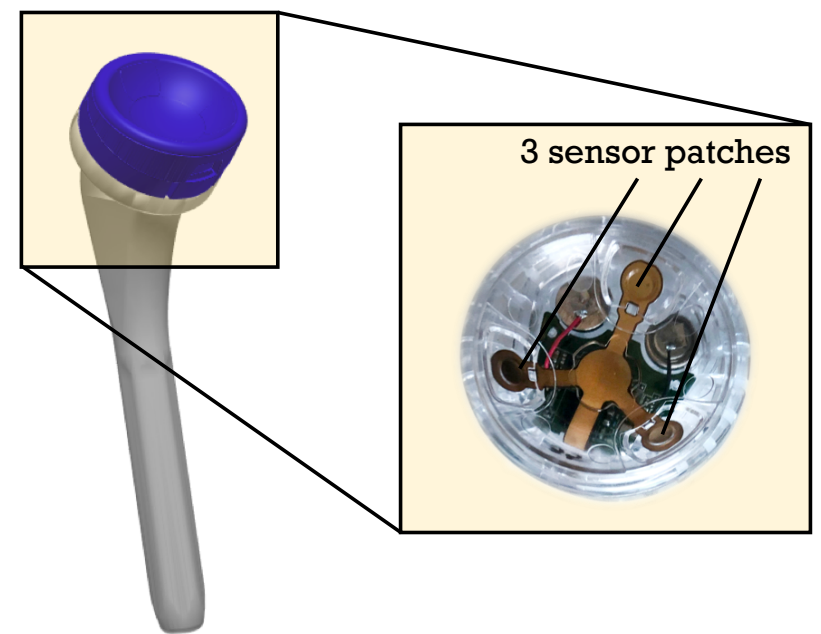

Figure 1: Load sensor for reverse Total Shoulder Arthroplasty (rTSA). 


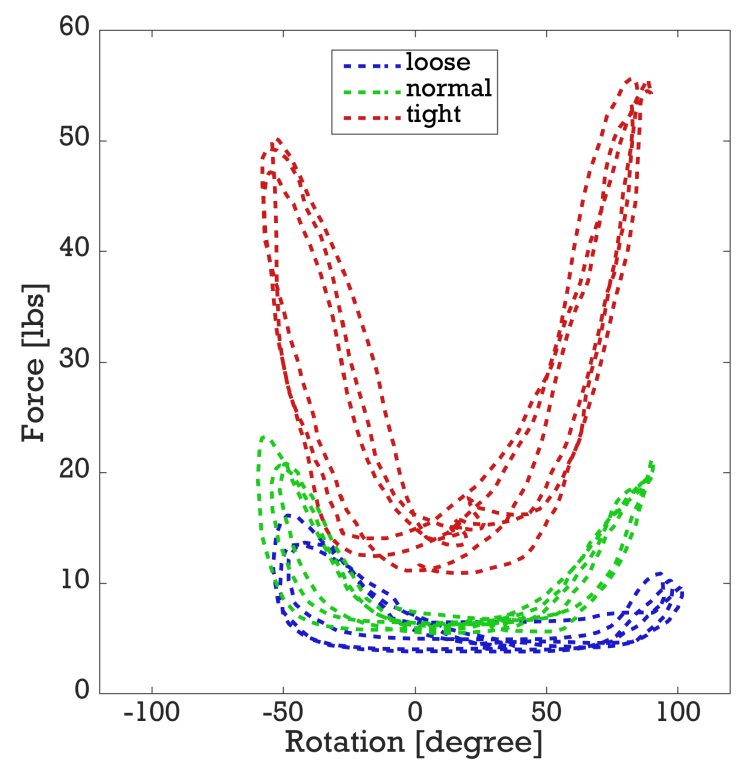

Figure 2: Load magnitude during range of motion for loose / normal / tight conditions.

\section{References}

Chae et al., Instability in Reverse Total Shoulder Arthroplasty, J Am Acad Orthop Surg. 2018 Sep 1;26(17):587-596. doi: 10.5435/JAAOS-D-16-00408.

Elmore et al., Soft tissue structures resisting anterior instability in a computational glenohumeral joint model, Comput Methods Biomech Biomed Engin. 2013;16(7):781-9. doi:10.1080/10255842.2011.641120. Epub 2012 Feb 2.

Higa M. et al., Instrumented Trial Prosthesis for Intraoperative Measurements of Joint Reaction Forces during Reverse Total Shoulder Arthroplasty, Sensors and Materials, Vol. 30, No. 9 (2018) 1989-1996.

Veeger et al., Shoulder function: the perfect compromise between mobility and stability, J Biomech. 2007;40(10):2119-29. Epub 2007 Jan 12.

Westerhoff et al., In vivo measurement of shoulder joint loads during activities of daily living, $\mathrm{J}$ Biomech. 2009 Aug 25;42(12):1840-9. doi: 10.1016/j.jbiomech.2009.05.035. Epub 2009 Jul 30. 